\title{
EDDY CURRENT NON-DESTRUCTIVE EVALUATION OF CONDUCTIVE MATERIALS
}

Non-destructive methods of material properties evaluation are presented in the paper. The methods are useful especially for increasing the safety of sophisticated and environment threatening facilities and, consequently, for decreasing the operating expenditures of those facilities. The attention is paid mainly to the method that utilises eddy currents induced in the surface layer of conducting samples by means of a small flat coil. The eddy current testing method is theoretically described and results of its particular application are presented in the paper.

\section{Introduction}

The present-day period is characteristic of a fast development of sophisticated technologies. The technologies strongly influence not only industry but also global living surroundings. Due to their price and possible consequences of their breakdown a permanent inspection of their integrity and function becomes more and more important. The inspection has many aspects and thus different approaches are utilised. A non-destructive testing (NDT) of materials properties plays an important role among them as a tested structure is not damaged during the inspection and usually there is no need for disassembling of the tested structure. Accordingly, the NDT is used for in-service inspection as well as for evaluation of manufacturing processes. Reliable inspection methods are very important because of two closely related aspects. The first and the most important one is the safety. Discovering structural anomalies and preventing accidents is the basic task of the NDT. The second aspect is economical because an effective utilisation and safe prolongation of life-span of expensive facilities such as nuclear reactors, dams, space devices, aircraft, submarines, sophisticated medical devices, information databases etc. can be based on a periodical monitoring of their health.

The most important task of the non-destructive evaluation consists in the discovering of different defects arisen during production or service of a component due to fatigue, overloading or influence of extreme conditions. Different methods are utilised for the purpose. It depends on a character of a material being tested as well as on a structure and a location of an expected defect which of the methods is the most reliable one. The methods differ mainly in resolution and sensitivity to various kinds of defects (e.g. surface or volume).

The methods of non-destructive evaluation can be divided into several main groups:

- Mechanical waves techniques - mechanical vibrations of an inspected body are investigated, namely, propagation of surface or volume mechanical waves in a sample or emission of acoustic waves from the body. Resolution of mechanical wave methods is limited by a wavelength or a frequency of the wave.

- Ultrasonic defectoscopy.

- Acoustic emission.

- Investigation of resonance spectra of mechanical vibrations.

- Electromagnetic waves techniques - interaction of an electromagnetic wave with an inspected body is evaluated, mainly propagation, reflection and scattering of the electromagnetic wave caused by non-homogeneities in a sample.

- Optical methods (e.g. microscopic investigation, holographic and interferometric methods) are very effective mainly in a case of investigation of a sample deformation or changes of quality of its surface.

- Infra-red thermography is used for investigation of non-homogeneous thermal profile of a sample caused by electrical or mechanical losses (e.g. heating of bearing or breaks, heating of electric joints or electric devices).

- X-ray investigation represents very effective and powerful means of defectoscopy. Influence of a sample structure on the $\mathrm{X}$-rays propagation through the sample is investigated. The method is sensitive to internal defects of conducting samples like welded joints, parts of motors, railway carriages wheels, organic samples, etc.

- Nuclear methods.

- Radioactive sampling method works based on addition of a radioactive substance (liquid or gas) into a flowing fluid and consequent dosimetric evaluation of the fluid propagation thorough an investigated system or penetration into investigated defects.

- Nuclear magnetic resonance is a method which is able to discover special atoms in a sample, e.g. hydrogen atoms of the water. The method is effectively used for the investigation of living structures (plants or animals).

- Computer tomography methods represent modern tools developed in connection with evolution of powerful computers. Tomography imaging consists in computer aided processing of a huge amount of data obtained by a successive detection of radiation emitted by an investigated body. A source of a signal might be

\footnotetext{
* L. Janoušek, T. Marek, D. Gombárska

Department of Electromagnetic and Biomedical Engineering, Faculty of Electrical Engineering, University of Žilina, Univerzitná 1, 01026 Žilina,

Slovakia, E-mail: janousek@fel.utc.sk, marek@fel.utc.sk, gombarska@fel.utc.sk
} 
X-rays, nuclear magnetic resonance signal, radioactive emission etc. The computer tomography is a tool for 3D-imaging of a sample internal structure.

- Electromagnetic inductive methods employ interaction between the alternating electromagnetic field with an electro-dynamic structure of a conducting material.

- Acoustic wave which propagates through a sample can be generated by an electromagnetic field in a surface conductive layer of the sample. A special probe used for generating and detecting of the wave is known as EMAT (ElectromagneticAcoustic Transducer). The method combines advantages of the ultrasonic inspection together with an advantage of a noncontact inductive method for the ultrasound generation and detection. Detailed description of the method together with some results of its development was published in [1 - 5].

- External electromagnetic field generates strongly attenuated electromagnetic wave known as the skin effect in the surface layer of a conducting sample. It is connected with existence of induced eddy currents. Non-destructive eddy current testing (ECT) is utilized for non-contact inspection of conducting bodies. This method is described in detail in [6 - 12] and in the present paper.

\section{Eddy Current Testing}

The principle of the ECT method underlies in the interaction of the induced eddy currents with a structure of an examined body. The alternating electromagnetic field generated by a suitable coil penetrates into a surface layer of the body to an effective depth given by an expression:

$$
\delta=\sqrt{\frac{2}{\omega \mu \sigma}},
$$

where $\omega$ is the angular frequency, $\mu$ and $\sigma$ are the permeability and the conductivity of a material, see Fig. 1.

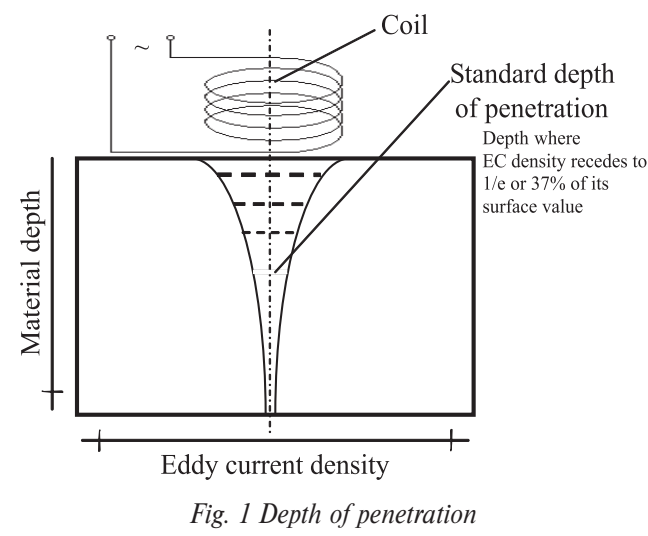

The eddy currents (EC) are influenced by defects and other non-homogeneities in the tested sample. The electromagnetic field generated by the EC can be detected by the same coil (self inductance ECT probe of the absolute type) and is evaluated as a change in the coil's impedance. The impedance is influenced not only by the sample properties but also by a configuration of the coil and by a lift-off distance of the coil from the sample surface. Evaluation of the obtained signal is a challenge of the method. A relation between the detected change in the impedance of the coil and a structure of the tested sample is very complex and has to be evaluated by means of special transformation procedures performed using dedicated computer codes (inverse problem) developed based on the forward simulations of ECT tasks.

The ECT method is effectively used only for the examination of surface breaking defects because the EC amplitude exponentially decays with the depth in the tested sample. A surface layer that can be effectively inspected by the ECT goes from several decimals of $\mathrm{mm}$ ( $100 \mathrm{kHz}$ range) up to several $\mathrm{mm}$ ( $\mathrm{kHz}$ range). Selection of the inspection frequency depends on an estimated character as well as on an expected depth of defects. On the other hand, the resolution of the method is limited by the electromagnetic wavelength, which is comparable with the effective skin depths $\lambda=2 \pi \delta$ and thus it is dependent on frequency. Usually multi-frequency examination is used during an inspection employing the ECT method.

The input impedance and thus the primary current of the excitation circuit are affected by material properties of a specimen, geometrical arrangement of a probe itself and the lift-off distance from the surface of a sample. When the last two parameters are kept constant during the inspection, the measured ECT signal depends on changes of the material properties in general and provides the information about the location of discontinuity, its shape and proportions.

The main advantages of the ECT method are [10]:

- sensitivity to small cracks and structural non-homogeneities;

- high resolution;

- versatility;

- inspection gives immediate results;

- portable equipment;

- non-contact inspection;

- inspection of complex structures.

On the other hand, the ECT has some limitations arising from its own physical principles. The primary limitation is given by applicability of the method to the inspection of conducting materials only. The EC depth of penetration is limited by the skin effect, therefore the method is applicable only for detection of surface and near subsurface defects or for the volumetric inspection of thin materials. Defects lying parallel to the coil's winding are hardly detectable. In addition, a surface roughness of a tested object influences the measured data and decreases the probability of detection as well as deteriorates the evaluation of the crack. However, because of the advantages listed above, the ECT is frequently used in many industrial fields. It is mainly applied in the non-destructive testing of various structural components. The ECT is also used for measurement of a thickness of conductive materials. For example, it can be used in evaluation of a wall thinning due to corrosion or in measurement of conductive material coating or paint- 


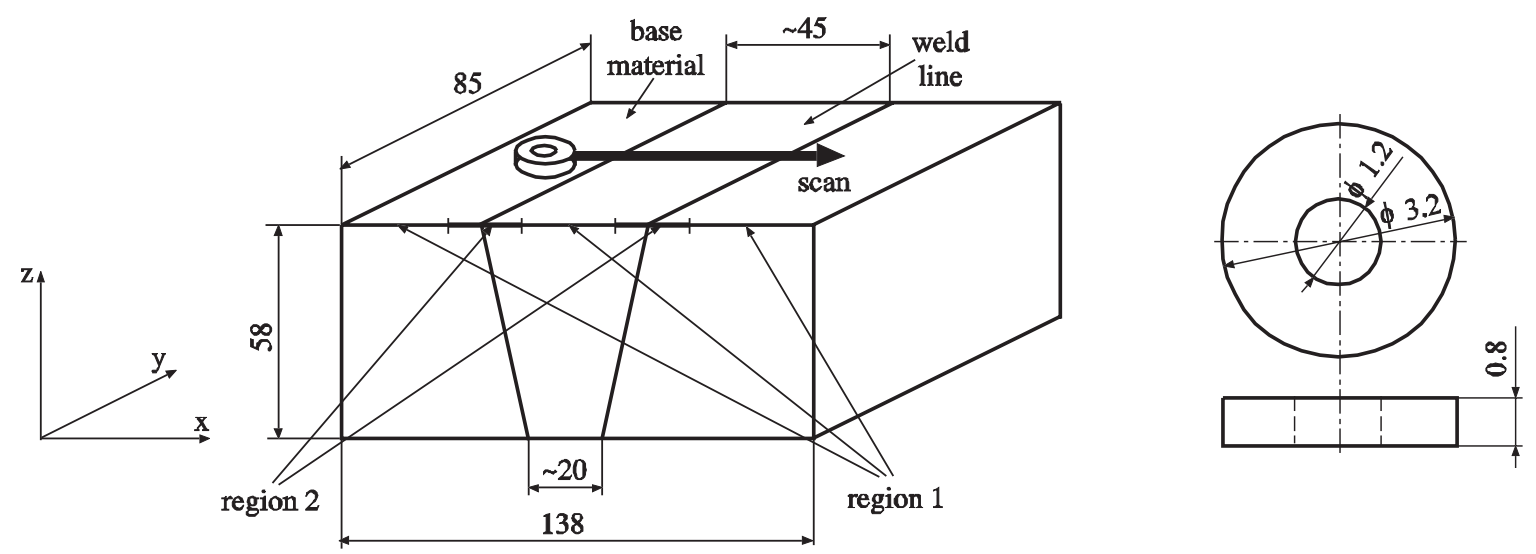

Fig. 2 Configuration and dimensions of the specimen and the pancake probe

ing thickness. The ECT is widely employed for an examination of the material properties during manufacturing process as well.

\section{Measurement of the conductivity using ECT}

This section deals with one of practical applications of the ECT; a measurement of conductivity is concerned here.

A welded specimen, shown in Fig. 2, is inspected in the study and its conductivity is estimated based on the measured values. The specimen base material is Alloy 600. The material is frequently used in structural components of nuclear power plants where the ECT is applied for non-destructive testing. The electromagnetic parameters, i.e. conductivity and relative permeability, of the base material are generally known. However, one of basic elements in such components is weld. The conductivity variation in the weld is unknown while the relative permeability is the same as the one of the base material, i.e. $\mu_{r}=1$. To be able to deal with such configuration in numerical simulations using the finite element method, a numerical model of the specimen with known parameters has to be built.

A circular coil, so called self-inductance absolute pancake probe, shown in Fig. 2, is used for this study. The probe scanned over the surface of the specimen as depicted in Fig. 2 across the weld line. A professional ECT instrument ASWAN was used to drive the probe and at the same time to pick-up the signal. The length of the scanning line was $90 \mathrm{~mm}$ and the probe moved with a lift-off of $0.25 \mathrm{~mm}$. The signals for three frequencies of 200,300 and $400 \mathrm{kHz}$ were gained at the same time and they were stored in a PC for further analyses. The real part and the imaginary part of the detected signal for the frequency of $200 \mathrm{kHz}$ are shown in Fig. 3 .

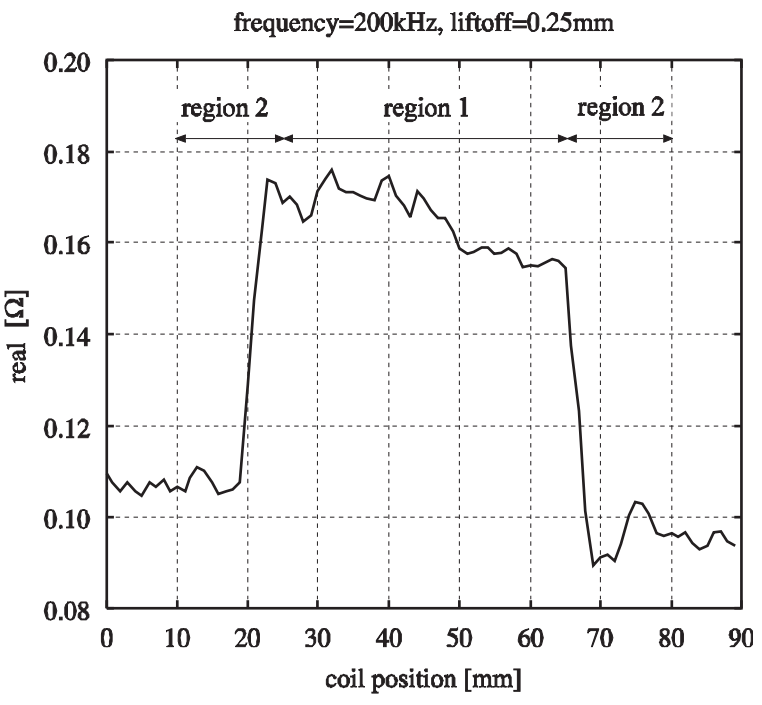

a)

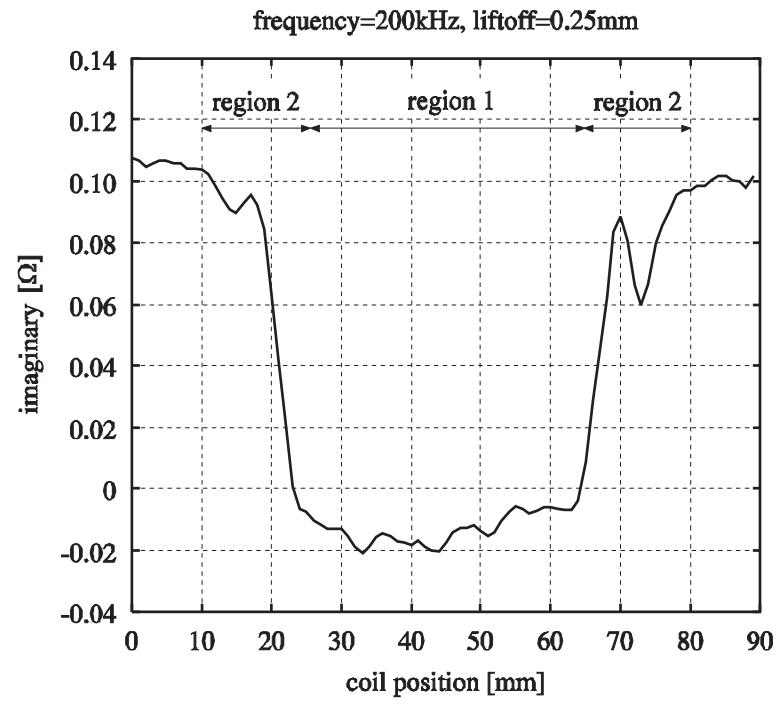

b)

Fig. 3 Detected signal across the weld line: (a) real part, (b) imaginary part. 


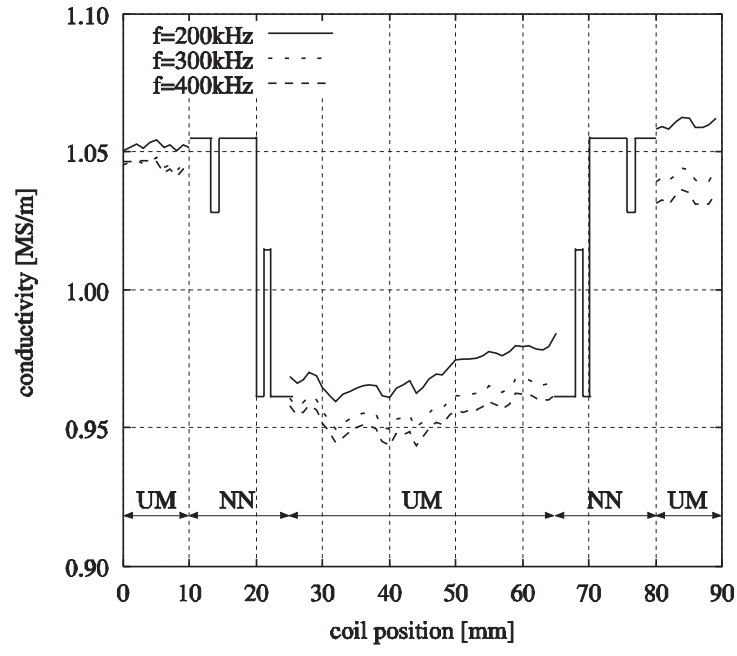

Fig. 4 The reconstructed profile of conductivity.

Two assumptions are made to reconstruct the conductivity variation across the weld line:

- The surface of welded specimen is divided into two regions. The first (Fig. 2 - region 1, part of the base material, middle part of the weld) is quasi stable one, where the signal variation is small. The second, transient one (Fig. 2 - region 2) covers a heat affected zone and the boundary of the weld, where the signal variation is large.

- The conductivity is supposed to vary just in one direction perpendicular to the weld line.

According to these assumptions, two different prediction methods have been proposed for the two regions separately [13] The conductivity distribution along the quasi stable regions is estimated based on the relationships derived from a uniform model. It was proved that such method can deal also with no uniform distribution of the conductivity if the conductivity variation is limited. A neural network approach is utilized to tackle the conductivity profile recognition along the transient regions. More description about the methods can be found in [13]. All the input data for both the approaches were acquired by numerical simulations using the finite element method.

The reconstructed profile of conductivity across the weld line is shown in Fig. 4. The conductivity in the quasi stable regions (region 1) was estimated based on the uniform model (UM) for all the three frequencies. As it can be seen the weld features the conductivity that is by approximately $7-8 \%$ lower than the one of the base metal. In the transient regions (region 2), the conductivity profile was estimated by using the neural network $(\mathrm{NN})$ for

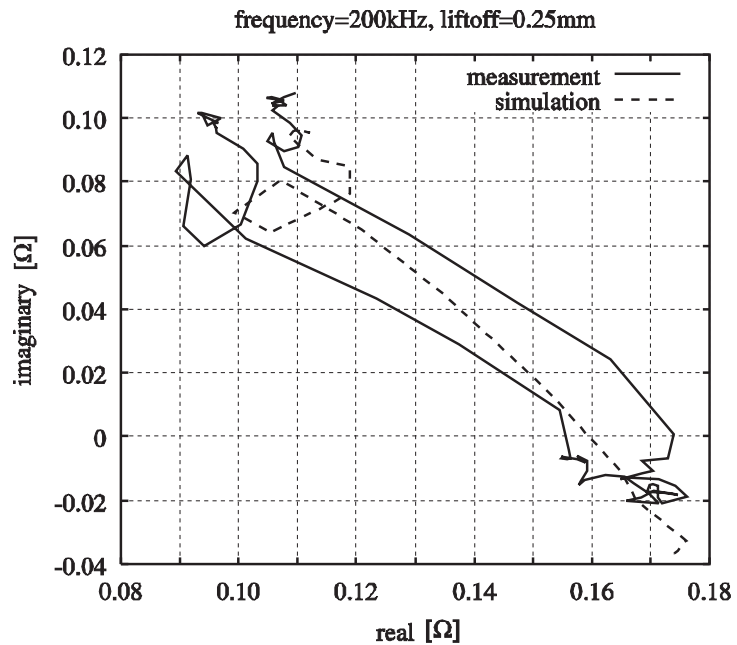

Fig. 5 Lissajous plot, comparison between the measured and simulated signals.

a frequency of $200 \mathrm{kHz}$. The conductivity changes sharply at the border of the weld line.

To prove the results of the conductivity profile reconstruction a finite element model of the target with taking into account the estimated profile of conductivity was built and the probe signal under the same conditions as the one used in the experiment was simulated. Both the signals, i.e. experimental and simulated ones, are shown in Fig. 5. The numerical results well coincide with the measured ones.

\section{Conclusion}

The importance of the non-destructive testing (NDT) of materials was emphasized in the paper. Different NDT methods were described focusing mainly on utilization of the eddy current phenomena in the non-destructive evaluation of conductive materials. The basic principle of the method was explained and possible application areas of its utilization were shown. The welded structure made of Alloy 600 was inspected by means of the eddy current NDT. The conductivity profile in the base material as well as in the weld was reconstructed from the measured data. It was found out that the conductivity of the weld is approximately by $8 \%$ lower than the one of the base material. The numerical simulations of the signal using the finite element method based on the reconstructed profile of conductivity were presented. The comparison of the measured and simulated signals confirmed the validity of the gained results.

\section{References}

[1] ČÁPOVÁ, K., ČÁP, I.: Electroacoustic Method of Thin Surface Layer Investigation, Advanced Computational Electromagnetics and Mechanics 9, IOS Press Ohmsha, 1995, ISSN 1383-7281, pp. 292-300. 
[2] ČÁPOVÁ, K., ČÁP, I.: Acoustically Excited Electromagnetic Wave in Metal. Studies in Applied Electromagnetics and Mechanics 13. IOS Press Ohmsha (Amsterdam, Berlin, Oxford, Tokyo, Washington D.C), 1998, ISSN 1383-7281, pp. 811-814.

[3] ČÁPOVÁ, K., ČÁP, I.: Material Anisotropy Investigation Using Anomalous Electromagnetic Generation of Acoustic Wave. Electromagnetic, Studies in Applied Electromagnetics and Mechanics 14, IOS Press Ohmsha (Amsterdam, Berlin, Oxford, Tokyo, Washington D.C.), 1998, ISSN 1383-7281, pp. 74-81.

[4] ČÁPOVÁ, K., ČÁP, I., FAKTOROVÁ, D.: Special Problems of Material Non-destructive Testing by Electromagnetic Acoustic Transducer. JSAEM Studies in Applied Electromagnetics and Mechanics, 9, 2001, Tokyo Japan, pp. 541-542.

[5] ČÁPOVÁ, K., ČÁP, I., FAKTOROVÁ, D.: Special Problems of Electromagnetic-acoustic Transducer for Material Non-destructive Testing, International Journal of Applied Electromagnetics and Mechanics,15, 2002, IOS Press, pp. 73-77.

[6] MAREK, T., FAKTOROVÁ, D., ČÁPOVÁ, K.: The Eddy Current Method using in Nondestructive Testing, In proceedings of Advanced Methods in Theory of Electrical Engineering. Plzeň, Czech Republic, Sept. 2005, ISBN 80-7043-392-2, pp. C13-C18.

[7] http://www.un.org/esa/sustdev (accessed on 2005/09/14).

[8] http://www.ndt.net/article/ecndt98/aero/028/028.htm (accessed on 2005/09/14).

[9] http://www.ndt.net/article/pacndt98/18/18.htm (accessed on 2005/09/15).

[10] http://www.ndt.net/article/wcndt00/papers/idn345/idn345.htm (accessed on 2005/09/15).

[11] http://www.ndt-ed.org/EducationResources/CommunityCollege/communitycollege.htm (accessed on 2005/09/16).

[12] AULD, B. A. AND MOULDER, J. C.: Review of Advances in Quantitative Eddy Current Nondestructive Evaluation, Journal of Nondestructive Evaluation, March 1999, Vol. 18, No. 1, Plenum Press, New York and London, ISSN 0195-9298, pp. 3-36.

[13] JANOUSEK, L. Et Al.: Recognition of INCONEL Weld Conductivity Variation by means of eddy current testing, Studies in Applied Electromagnetics and Mechanics, Vol. 24, 2004, IOS Press, ISSN 1383-7281, pp. 286-293.

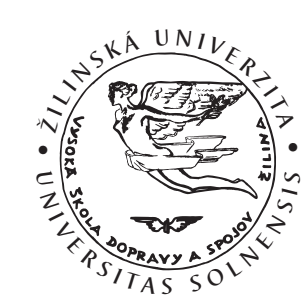

\section{CRISES SITUATIONS SOLUTION IN SPECIFIC ENVIRONMENT}

\section{The Eleventh International Scientific Conference} 29 and 30 June 2006

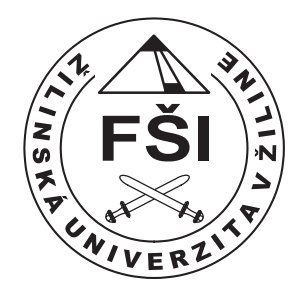

We would like to inform you that the Faculty of Special Engineering of the University of Žilina in Žilina is organizing an international scientific conference called Crisis Situations Solution in Specific Environment.

The goal of the conference is to exchange the latest findings and practical experience of crisis management, persons and property protection and the tasks of human factors in crises situations.

Conference sections:

Section No.1:

Crisis Management and National Security

Section No.2:

Security management - people and property protection

Section No.3:

Solution of Economical Crises

Section No.4:

Human factor in crisis management

Section No.5:

Transport in Crisis Situations

For further information please visit our web page http://fsi.utc.sk/kkm/ or contact our secretary of the conference on e-mail: crisis@fsi.utc.sk or by phone: +421 415136748. 